\title{
Testing non-classical theories of electromagnetism with ion interferometry
}

\author{
B. Neyenhuis, D. Christensen, and D. S. Durfee \\ Brigham Young University, Department of Physics and Astronomy, Provo, UT 84602
}

(Dated: 19 September 2007)

\begin{abstract}
We discuss using a table-top ion interferometer to search for deviations from Coulomb's inversesquare law. Such deviations would result from non-classical effects such at a non-zero photon rest mass. We discuss the theory behind the proposed measurement, explain which fundamental, experimentally controllable parameters are the relevant figures of merit, and calculate the expected performance of such a device in terms of these parameters. The sensitivity to deviations in the exponent of the inverse-square law is predicted to be a few times $10^{-22}$, an improvement by five orders of magnitude over current experiments. It could measure a non-zero photon rest mass smaller than $9 \times 10^{-50}$ grams, nearly 100 times smaller than current laboratory experiments.
\end{abstract}

The experimental search for deviations from current theories will eventually lead to the next, more fundamental theory of physics. Such studies challenge the Standard Model and give insight into the form of the underlying, more elemental theory. Coulomb's inverse-square law is the foundational law in electrostatics. Gauss's Law and Maxwell's equations are built upon this law and the principle of superposition. Precision tests of this law are essential to push forward our understanding of electromagnetism and its relation to the other forces.

Detection of any deviation from Coulomb's law would have far-reaching implications. Maxwell's equations and much of the Standard Model would have to be modified. The notion that absolute electrostatic potential is arbitrary would have to be abandoned, along with many other tenets of classical electromagnetism. Inversesquare-law violation would suggest a finite range for the electromagnetic force, implying a non-zero photon rest mass [1, 2, 3]. Consequences of massive photons include a frequency-dependent velocity of light in vacuum and electromagnetic waves with a longitudinal component of polarization [4]. Several grand-unification theories include massive photons [5, [6], and further tests of the inverse-square law can help confirm or disprove them.

Several studies have searched for consequences of massive photons rather than testing the inverse-square law directly [2]. These studies involve many assumptions about the nature of interstellar space and the sources of the measured light waves. It is therefore necessary to verify these results with laboratory experiments where variables can be better controlled [20]. And while the possibility of a massive photon supplies additional motivation and provides a common parameter to compare experiments, it is possible that Coulomb's law is violated for reasons unrelated to photon rest mass. Only an experiment which specifically measures the inverse-square law would be sensitive to these effects.

Although Coulomb's law has been tested many times over the last two and a half centuries [3, $7,8,8,9,10,11]$, this subject has seen little progress in the last three decades. The smallest laboratory-based limit on the photon rest mass was reported 24 years ago [11]. In this experiment an alternating voltage was applied between two conducting shells, and the induced voltage between

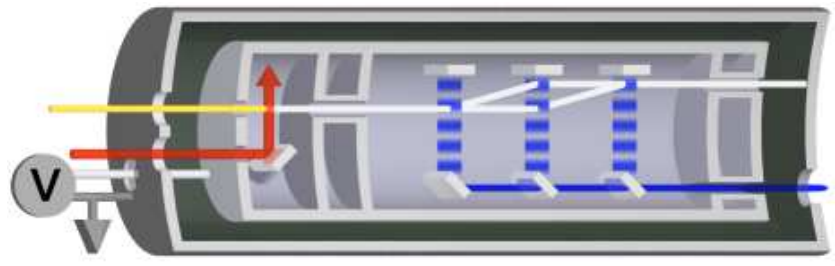

FIG. 1: (Color online) A cut-away cartoon of the proposed experiment. The diagram is not to scale, and some dimensions have been greatly exaggerated for visibility.

the outer of the two and a third shell was measured with solid-state electronics. This measurement improved upon the best previous measurement, 12 years old at the time [10], by only a factor of 2.5 . In this paper we show that it should be possible to revitalize this key field of study and improve sensitivity by orders of magnitude using a new approach - charged particle matter-wave interferometry.

In the proposed experiment, a possible Coulomb's-law violating electric field inside of a conducting shell is measured with an ion interferometer. As shown in Fig. 1, ions travel through a conducting cylinder nested inside of a second cylinder. The outer conductor is grounded, and a time-varying voltage is applied to the inner conductor. A slow beam of atoms passes through small holes in the conductors. The atoms are ionized with a laser beam, shown as an arrow in the figure, and pass through three gratings to form a Mach-Zehnder interferometer. If an electric field is present in the inner conductor, the two interferometer arms will pass through different potentials, resulting in a relative phase shift. Using optical gratings would allow state-selective readout and avoid drawbacks of physical gratings [12], including charge build-up and image charges in the gratings. Using Raman transitions [13] would allow precise control of grating phases.

To calculate the sensitivity that could be achieved, we start with a modified version of Laplace's equation derived from the Proca action for massive photons:

$$
\nabla^{2} \phi-\mu_{\gamma}^{2} \phi=0
$$




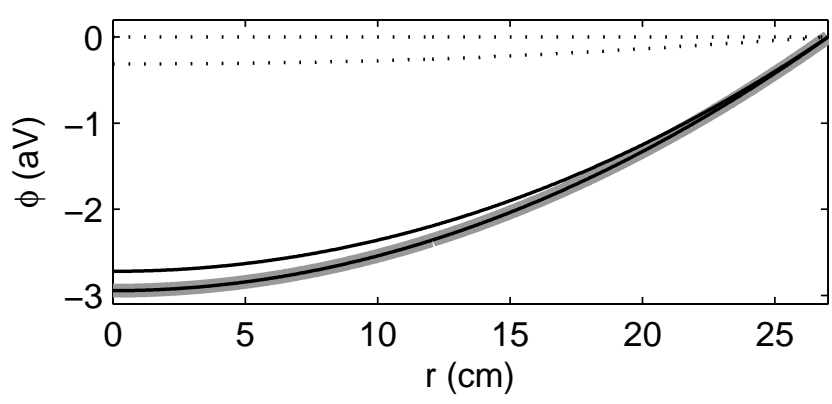

FIG. 2: Calculations of potentials in a $2.6 \mathrm{~m} \mathrm{long,} 27 \mathrm{~cm}$ radius tube held at $200 \mathrm{kV}$. The calculation assumes $m_{\gamma}=$ $1 \times 10^{-50}$ grams. Potentials are plotted vs. the radial distance from the tube axis. The black lines are the deviation from the classical potential at axial distances of zero (lower line) and one meter (upper line) from the middle of the tube, plotted on top of a thick gray line representing the deviation for an infinite tube. The dotted lines show the calculated classical fringing-field potentials at the same locations multiplied by $10^{35}$ to make them visible on this scale.

In this equation $\phi$ is the scalar electrostatic potential, and $\mu_{\gamma}=m_{\gamma} c / \hbar$, where $m_{\gamma}$ is the photon rest mass, $\hbar$ is Planck's constant divided by $2 \pi$, and $c$ is the canonical speed of light in vacuum.

In the limit as $\mu_{\gamma} \rightarrow 0$, Eq. 1 becomes Laplace's equation. For a spherically symmetric system, Laplace's equation has the familiar solutions $\phi(r)=A / r$ and $\phi(r)=B$, where $A$ and $B$ are constants. The $A / r$ solution is the classical point-charge potential. The constant $B$ solution allows us to arbitrarily define a point to be at zero potential without changing the fields described by the potential. If $\mu_{\gamma} \neq 0$, the solutions for a spherically symmetric system are a Yukawa potential $\phi(r)=(A / r) \exp \left(-\mu_{\gamma} r\right)$, and an exponentially growing solution $\phi(r)=(B / r) \exp \left(\mu_{\gamma} r\right)$. The Yukawa-potential solution lets us interpret $1 / \mu_{\gamma}$ as an effective range of the Coulomb force. Without a constant solution, absolute potential has physical significance and we are no longer free to arbitrarily choose where $\phi$ equals zero.

Due to the elongated geometry of the proposed experiment, we will approximate the finite inner conductor with an infinitely long tube. Numerical and analytical studies have verified that this is a good approximation for reasonably long tubes (see Fig. 2 and 14]). For a system with no angular or longitudinal dependence, solutions to Eq. 1 are the zeroth-order modified Bessel functions. Applying the conditions that $\phi(r)$ must equal the applied voltage when $r=R$ (the radius of the tube), we determine that to lowest order in $\mu_{\gamma}$ the potential inside the inner tube is given by

$$
\phi(r) \approx\left(V+V_{g}\right)\left[1+\frac{\mu_{\gamma}^{2}}{4}\left(r^{2}-R^{2}\right)\right] .
$$

Here $V$ is the voltage applied to the inner tube relative to the outer tube, and $V_{g}$ is the unknown voltage of the outer, grounded tube.

Rather than absolute potential, the interferometer will measure the potential difference between the two arms. Each of the arms in Fig. 1 consists of one horizontal and one diagonal segment. The diagonal segments can be neglected because they both pass through identical potentials inducing equal phase shifts. The horizontal segments, however, travel through different potentials. Assuming that the two horizontal segments are a distance $r_{0}$ and $r_{0}+s$ from the center of the tube, the potential difference between them is

$$
\Delta \phi=\phi\left(r_{0}+s\right)-\phi\left(r_{0}\right) \approx \frac{\mu_{\gamma}^{2}}{4}\left(V+V_{g}\right)\left(s^{2}+2 r_{0} s\right) .
$$

If $\tau$ is the time that it takes the ions to travel the length of the horizontal segments, and $e$ is the ion charge, the interferometer phase $\Phi$ is given by

$$
\Phi \approx \frac{e \mu_{\gamma}^{2}}{4}\left(V+V_{g}\right)\left(s^{2}+2 r_{0} s\right) \frac{\tau}{\hbar}+\Phi_{0},
$$

where $\Phi_{0}$ is the phase when $V+V_{g}=0$. This term includes all phase shifts which are not dependent on the absolute potential, such as those due to patch charges, imbalanced interferometer arms, etc. Although $V_{g}$ and $\phi_{0}$ are unknown, one could change the potential $V$ by an amount $\Delta V$ and look for a correlated change in the interferometer phase. Because the $\sim 700 \mu \mathrm{F}$ capacitance of the Earth is very large compared to the $\sim 1.6 \mathrm{nF}$ capacitance of the proposed conductors, $V_{g}$ will remain roughly constant as $V$ is changed, and the difference in phase due to the potential change will be

$$
\Delta \Phi \approx \frac{e \Delta V \mu_{\gamma}^{2} \tau}{4 \hbar}\left(s^{2}+2 r_{0} s\right) .
$$

Solving Eq. 5 for $\mu_{\gamma}$ we can determine the rest mass of the photon from the measured interferometer phase shift:

$$
m_{\gamma} \approx \frac{\hbar}{c}\left[\frac{4 \hbar \Delta \Phi}{e \Delta V\left(s^{2}+2 r_{0} s\right) \tau}\right]^{1 / 2} .
$$

To estimate the smallest detectable $m_{\gamma}$, it is useful to rewrite Eq. 6 in terms of experimentally accessible parameters. One important parameter is the velocity of the ions $v$. A smaller velocity results in larger diffraction angles but more deflection by stray electric fields. Fields will be extremely small inside the tube at the locations of the gratings (see Fig. 22), but they could be much larger in the region where the ions are generated. If we write the velocity as $v=(2 \mathrm{eV} / \mathrm{m})^{1 / 2}$ where $m$ is the mass of the ions and $V_{s}$ is the voltage which would just bring the ions to a stop, we can set $V_{s}$ to be several times the level of the expected stray fields to be sure that the trajectory of the ions is not greatly perturbed by them.

Two other important parameters are the maximum excursion of the ions from the center of the tube, $a=r_{0}+s$, and the distance between gratings, $L$. A larger tube radius accommodates a larger separation $s$ and offset $r_{0}$. 
A larger grating separation $L$ means that the ions will interact with the field longer $(\tau=L / v)$ and results in a greater separation of the two arms of the interferometer ( $s \approx L h / m v d$, where $h$ is Planck's constant and $d$ is the grating period). With these parameters in mind, we can rewrite Eq. 6 as

$$
m_{\gamma} \approx \frac{\hbar}{c L}\left[\frac{2 \Delta \Phi d V_{s}}{\pi \Delta V a(1-Q)}\right]^{1 / 2}
$$

where the parameter $Q=s / 2 a=\pi L \hbar /\left(2 e m V_{s} a^{2} d^{2}\right)^{1 / 2}$ shows very weak dependence on ion mass and charge although higher charge and lower mass results in more precision for a given ion velocity, this is offset by the greater velocity needed to overcome deflections by stray fields. For arbitrary experimental parameters, $0<Q \leq$ $1 / 2$. For the parameters selected below, $Q$ is small for all possible ion masses, ranging from $1 \times 10^{-2}$ for ${ }^{1} \mathrm{H}^{+}$to $1 \times 10^{-3}$ for ${ }^{133} \mathrm{Cs}^{+}$, and the precision of the experiment will not change much with the mass or charge of the ion.

There are practical limits on $L$ and $a$ for a table-top apparatus. We chose $L$ to be one meter, and limited $a$ to be a conservative $25 \mathrm{~cm}$. For our numerical calculations (Fig. 22) we assumed a total length for the inner tube plus end-caps of $3 \mathrm{~m}$, and a tube radius of $27 \mathrm{~cm}$. This gives sufficient space to limit fringing fields, to be sure that the infinite-tube calculation is a good approximation in the region of the interferometer, and to keep the ions in the interferometer away from the tube surface. Only $a$, and not the outer radius of the tube, affect the precision predicted in Eq. 7, So a tube with a larger radius could be used to further limit ion-surface interactions without changing the predicted precision.

We assumed a grating period of $200 \mathrm{~nm}$, about half the wavelength of a readily-available uv diode laser. We selected a value of $400 \mathrm{kV}$ for $\Delta V$ because $\pm 200 \mathrm{kV}$ is within the range of what is possible with off-the-shelf power supplies and vacuum feed-throughs. Based on work done with atom interferometers [15], it should be possible to detect phase-shifts as small as $10^{-4}$ radians. We set the final parameter, $V_{s}$, to $0.5 \mathrm{mV}$ assuming that voltages due to stray fields can be controlled well below this level.

Given the assumed parameters the separation $s$ would range from $6.4 \mathrm{~mm}$ (for ${ }^{1} \mathrm{H}^{+}$) to $0.56 \mathrm{~mm}$ (for ${ }^{133} \mathrm{Cs}^{+}$), and the ion beam would enter the apparatus at a radius $r_{0}$ ranging from $24.4 \mathrm{~cm}\left({ }^{1} \mathrm{H}^{+}\right)$to $24.9 \mathrm{~cm}\left({ }^{133} \mathrm{Cs}^{+}\right)$. For electrons these parameters yield an $s$ larger than the radius of the tube, making electrons a poor choice for these experimental parameters. For a horizontal apparatus in gravity, assuming that the ions undergo a parabolic trajectory with the peak at the location of the center grating, the ions will fall a vertical distance ranging from $51 \mu \mathrm{m}$ $\left({ }^{1} \mathrm{H}^{+}\right)$to $6.8 \mathrm{~mm}\left({ }^{133} \mathrm{Cs}^{+}\right)$, giving them a vertical velocity of only $1.0 \times 10^{-4}\left({ }^{1} \mathrm{H}^{+}\right)$to $1.4 \times 10^{-2}\left({ }^{133} \mathrm{Cs}^{+}\right)$times their longitudinal velocity. The phase shift due to gravity will be constant as the applied voltage is changed, and will not affect the measurement.
The velocity of the ions, determined by $V_{s}$ and the mass of the ion, ranges from $311 \mathrm{~m} / \mathrm{s}\left({ }^{1} \mathrm{H}^{+}\right)$to $27 \mathrm{~m} / \mathrm{s}$ $\left({ }^{133} \mathrm{Cs}^{+}\right)$. Ions at these velocities could be generated by ionizing a slow neutral-atom beam; a velocity of $27 \mathrm{~m} / \mathrm{s}$ is a reasonable velocity for a beam of atoms from an LVIS source [16]. Higher velocities are easily obtained by accelerating the ions with a small potential. As such, any atom that can be laser cooled could be used, all resulting in similar sensitivity. However, lighter ions have the advantage of faster transit, which would make it possible to modulate the voltage applied to the tube at a higher frequency, reducing the effective bandwidth of systematic drifts.

With these parameters we predict a sensitivity to photon rest mass of $9 \times 10^{-50}$ grams, nearly two orders of magnitude smaller than the limit in 11]. In addition to photon-rest-mass limits, following the tradition of Cavendish [8] it is also common to assume that the point-charge potential falls off as $r^{-(1+\delta)}$ and to quantify inverse-square-law violations with the small parameter $\delta$. Because the Proca treatment isn't necessarily correct, this additional figure of merit is valuable. Unfortunately, the $r^{-(1+\delta)}$ potential does not come from an underlying theory. If such a theory existed, $r^{-(1+\delta)}$ would enter naturally as a solution to a modified version of Laplace's equation. At least one more solution, one which is finite at $r=0$, must exist. Knowing just one of the solutions is not sufficient to determine charge distributions.

It appears that previous experiments calculated limits on $\delta$ by integrating the point-charge potential over the classical charge distribution. But the unmodelled deviation of the true charge distribution from the classical distribution could greatly affect the magnitude of $\delta$. Furthermore, if the unknown equation is nonlinear, the potential cannot be related to an integral of point charges. It is also disturbing that in this formalism the units of the permittivity $\epsilon_{0}$ depend on $\delta$. Ignoring these concerns, we integrated the point-charge potential over the classical charge distribution and predict a limit on $\delta$ of a few times $10^{-22}$ in the proposed apparatus, an improvement of five orders of magnitude over the value reported in [11].

In addition to higher sensitivity, the proposed device overcomes a potential pitfall present in the most recent experiments. In these studies 10, 11] a voltage between two conducting shells was measured electronically. Any non-zero electric field would tend to draw a charge through the electronics to cancel the field. If 0.001 times the charge of an electron passed through the probe electronics, it would cancel the field due to a photon mass larger than the reported precision. But in our scheme the only influence the ions have on the system under test is the well-understood induction of an image charge in the conductor.

The largest errors in the proposed measurement are expected to be due to inertial-force shifts [15] and ionion interactions. The ion-ion interactions can be reduced by limiting the number of ions inside the conductor at 
any given time - in the limit of a single ion at a time, this effect disappears while still affording a count rate of tens to hundreds of ions per second. This drift can also be reduced by using non-classical, anti-bunched ion beams.

We performed numerical calculations and piecewise analytical solutions to verify that fringing fields from holes in the conductor could be made negligibly small [14]. The calculations show that fringing fields will be tens of orders of magnitude below the detection limit (see Fig. 2), and should not be an issue. In these calculations the inner conductor was capped with $20 \mathrm{~cm}$-long end caps to reduce fringing fields. Because the calculations assumed axial symmetry, the holes in the end cap were replaced with ring-shaped apertures. As such, the calculations greatly overestimate the size of the fringing fields.

Drifts in patch charges [17] and similar effects should not be correlated with changes in the applied voltage, especially considering the extremely small level of the fringing fields. The susceptibility to stray electric fields should be no greater than in other recent laboratory tests of Coulomb's law, and in 11] it was implied that these effects were not a limitation. Magnetic shielding will be necessary, and magnetic fields created by the charging and discharging of the conductors will have to be taken into account. The effect of eddy currents could be reduced exponentially by increasing the time between voltage reversals. And although static fields will not affect the phase difference, large static fields will reduce fringe contrast. Nevertheless, these difficulties are surmountable in a reasonable experiment.

In conclusion, we have discussed the prospect of using ion interferometry to search for violations of Coulomb's law. Calculations using reasonable parameters suggest that a table-top device should be able to detect a photon rest mass at the level of $9 \times 10^{-50}$ grams, and measure deviation in the exponent of Coulomb's inverse-square law at the level of a few times $10^{-22}$, both representing an improvement of several orders of magnitude over current laboratory measurements. In addition, the apparatus would be immune to effects related to the modification of the field by the instrument used to measure it.

We acknowledge Ross Spencer for his assistance with numerical calculations. This work was funded by BYU's Office of Research and Creative Activities.
[1] A. S. Goldhaber and M. M. Nieto, Rev. Mod. Phys. 43, 277 (1971).

[2] L.-C. Tu, J. Luo, and G. T. Gillies, Rep. Prog. Phys. 68, 77 (2005).

[3] J. D. Jackson, Classical Electrodynamics (Wiley, New York, 1975), pp. 5-9, 597-601, 2nd ed.

[4] W. Greiner and J. Reinhardt, Field Quantization (Springer, 1996).

[5] N. Arkani-Hamed, S. Dimopoulos, and G. Dvali, Phys. Lett. B 429, 263 (1998).

[6] V. A. Kostelecky and S. Samuel, Phys. Rev. Lett. 66, 1811 (1991).

[7] R. S. Elliott, Electomagnetics (McGraw-Hill, New York, 1966), pp. 100-101.

[8] H. Cavendish, The Electrical Researches of the Honourable Henry Cavendish (Cambridge University Press, Cambridge, 1879), pp. 104-13.

[9] J. C. Maxwell, A Treatise on Electricity and Magnetism 3rd edn (Dover, 1873).

[10] E. R. Williams, J. E. Faller, and H. A. Hill, Phys. Rev. Lett. 26, 721 (1971).

[11] R. E. Crandall, Am. J. Phys. 51, 698 (1983).

[12] G. Gronniger, B. Barwick, H. Batelaan, T. Savas,
D. Pritchard, and A. Cronin, Appl. Phys. Lett. 87, 124104 (2005).

[13] T. L. Gustavson, P. Bouyer, and M. A. Kasevich, Phys. Rev. Lett. 78, 2046 (1997).

[14] D. Christensen, B. Neyenhuis, and D. S. Durfee, arXiv:physics/0609128 (2006).

[15] T. L. Gustavson, A. Landragin, and M. Kasevich, Class. Quantum Grav. 17, 2385 (2000).

[16] Z. T. Lu, K. L. Corwin, M. J. Renn, M. H. Anderson, E. A. Cornell, and C. E. Wieman, Phys. Rev. Lett. 77, 3331 (1996).

[17] L. Deslauriers, S. Olmschenk, D. Stick, W. K. Hensinger, J. Sterk, and C. Monroe, Phys. Rev. Lett. 97, 103007 (2006).

[18] J. Luo, L.-C. Tu, Z.-K. Hu, and E.-J. Luan, Phys. Rev. Lett. 90, 081801 (2003).

[19] R. Lakes, Phys. Rev. Lett. 80, 1826 (1998).

[20] The measurements in [18, 19] are sometimes erroneously considered laboratory experiments. But to obtain the photon rest mass from this type of measurement the cosmic vector potential must be guessed, making the experiment more like model-dependent astronomical studies. 